\title{
Amplification and modelling of bioaffinity detection with terahertz spectroscopy
}

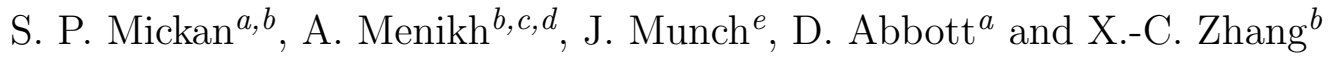 \\ ${ }^{a}$ Centre for Biomedical Engineering (CBME) and Department of Electrical \& \\ Electronic Engineering, The University of Adelaide 5005, Australia. \\ ${ }^{b}$ Center for Terahertz Research and Department of Physics, Applied Physics \& \\ Astronomy, Rensselaer Polytechnic Institute, Troy NY 12180, USA. \\ ${ }^{c}$ Department of Physics, The University of Constantine, Constantine 25000, Algeria. \\ ${ }^{d}$ Wadsworth Center, New York State Dept. of Health, Albany, NY 12201, USA. \\ ${ }^{e}$ Department of Physics \& Mathematical Physics, The University of Adelaide 5005, \\ Australia.
}

\begin{abstract}
A new bioaffinity sensor based on pulsed terahertz (THz) spectroscopy is able to sensitively detect the presence of ultra-thin bound biomolecular layers. The protein avidin and lipid biotin are noted for their very high binding affinity, and the ease for which they can be attached to residues with importance in many biosensing applications. We demonstrate the sensitivity of the pulsed $\mathrm{THz}$ spectrometer to thin avidin layers and to avidin amplified with micron-sized agarose beads. The experimental results can be simply modelled by considering transmission of the $\mathrm{THz}$ radiation at the thin film interfaces. We detect less than $10.3 \mathrm{ng} / \mathrm{cm}^{2}$ avidin, giving the THz system a detection capability of sub-thin solid films better than ellipsometry and reflectometry techniques.
\end{abstract}

Keywords: differential THz time-domain spectroscopy, biosensor, biotin-avidin

\section{INTRODUCTION}

There is a need for a new non-invasive biosensor technology in health care, food monitoring and weapon detection. Optical detection techniques have been used for more than a decade in the analytical laboratory since they provide a possible means of meeting such needs. The most useful techniques for monitoring on-off binding in the far-infrared are attenuated total internal reflection Fourier transform infrared (ATR-FTIR) spectroscopy and Raman spectroscopy. ${ }^{1,2}$ Unfortunately, the complications associated with cryogenic detectors in FTIR, and Rayleigh lines in Raman spectroscopy are among the anomalies that hamper FTIR and Raman spectroscopy from being widely used. The most successful optical biosensor in the market place is based on surface plasmon resonance (SPR). ${ }^{3,4}$ Recently a new detection method based on terahertz wave operating on the same principal as SPR has been proposed, and used successfully to detect minute amounts of label-free DNA molecules. ${ }^{5}$ This approach uses integrated $\mathrm{THz}$ waveguides, incorporating resonant $\mathrm{THz}$ structures. ${ }^{6,7}$ Using the newly-developed $\mathrm{THz}$ system Bolívar et al. achieved femtomole sensitivity to label-free DNA hybridization. This approach is still in progress and opens up new avenues for label-free detection. On the other hand, free-space configurations avoid the need for hardware modification, the difficulty of interconnecting a large number of resonators, simultaneous detection of thousands of genomes in a single experiment,

S. P. Mickan, Email: spmickan@eleceng.adelaide.edu.au; A. Menikh, Email: abdellah_menikh@yahoo.com; J. Munch, Email: jmunch@physics.adelaide.edu.au; D. Abbott, Email: dabbott@eleceng.adelaide.edu.au; X.C. Zhang, Email: zhangxz@rpi.edu. 
and delicate sample handling procedures. We have developed a new biochemical methodology, based on agarose beads, that can achieve higher sensitivity without hardware modification. By conjugating agarose particles to avidin and applying the conjugate to half of a biotin-coated quartz surface, a clear $\mathrm{THz}$ difference signal between biotin and the biotin-avidin complex is detected. We are able to detect less than $10.3 \mathrm{ng} / \mathrm{cm}^{2}$ avidin on the quartz surface, indicating the potential capability of this newly-developed method for future applications to detect DNA hybridization and antigen-antibody interactions. ${ }^{5,8}$

Avidin is a protein, which is comprised of four identical subunits, each binding one biotin molecule. The binding affinity between avidin and biotin is so high $\left(K_{a}=10^{15} \mathrm{~mol}^{-1}\right)$ that the formation of this complex can be regarded as nearly irreversible, on a scale comparable to covalent bonding. ${ }^{9}$ The high binding affinity of this system has found many applications, for example in affinity chromatography, in attaching antibodies to solid surfaces, precipitating liposomes, or targeting cells with liposomes. ${ }^{10,11}$ Importantly, it has been shown that biotin can be adsorbed to a hydrophobic surface without losing its specificity towards avidin, leading to the possibility of studying ligand-analyte interactions on supported lipid membranes.

This paper explores the $\mathrm{THz}$ signal from a previously affinity-bound compound in a lipid membranelike environment, and shows it can be modelled from a simple understanding of dielectric layers. This biosensor methodology is simple, non-invasive, inexpensive and does not require hardware modification in a typical $\mathrm{THz}$ spectrometer.

\section{SAMPLE PREPARATION}

The following chemicals were used as obtained without further purification: octadecanol, bovine serum albumin, biotin, and agarose beads, with an average diameter of $40 \mu \mathrm{m}$, conjugated to avidin from Sigma (St. Louis, MO). Quartz microscope slides $(25 \times 25 \mathrm{~mm})$ were obtained from Electron Microscopy Sciences (Washington, PA), and tested for thickness homogeneity before use. All reagents were HPLC grade and used as received. Aqueous solutions were prepared in double-distilled deionized water.

Prior to deposition, the quartz slides are cleaned in $50 \%$ hot nitric acid for an hour, and then rinsed thoroughly in double distilled water. The quartz slides are further subjected to $1 \mathrm{mg} / \mathrm{ml}$ octadecanol solution for half an hour. The approach that we use to immobilise a hydrophobic substrate on a hydrophilic surface relies on systematically controlling the interfacial region of the biosensor chip using a process widely referred to as molecular self-assembly. The spontaneous organization of the biotin lipid into the octadecanol self-assembled bilayers guarantees a certain level of stability to the octadecanol-biotin complexes. After drying and washing with double distilled water, the sample is dipped in $0.5 \mathrm{mg} / \mathrm{ml}$ biotin, dissolved in chloroform/methanol (5:1), and allowed to incubate for 45 minutes. Immersion in a solution of biotin results in an increase in thickness of $5 \mathrm{~nm} / \mathrm{cm}^{2}$, caused by the biotin interaction with octadecanol through hydrophobic interaction. Lastly, to eliminate the effect of non-specific interactions, the quartz slides are further incubated in $1 \%$ bovine serum albumin, then dried and washed several times with phosphate buffer solution.

The target molecules are then applied to one side of the biosensor slide. Either $10.3 \mathrm{ng}$ of avidin in $200 \mu \mathrm{l}$ double-distilled water, or avidin conjugated to agarose beads, is spread on $1 \mathrm{~cm}^{-2}$ of the quartz surface modified with biotin, and allowed to incubate for half an hour. After drying, the sample is washed with double-distilled water to remove unbound molecules. The only remaining molecules form a thin layer of tightly-bound avidin, or the avidin conjugate.

\section{THZ SPECTROMETER}

The double-modulated differential THz time-domain spectroscopy (DTDS) system used in this work has been described in more detail in our previous paper, ${ }^{8}$ and is schematically represented in Fig. 2. Briefly, the femtosecond laser source generates 150 -fs pulses at $86 \mathrm{MHz}$ and $1.5 \mathrm{~W}$ of average power. 


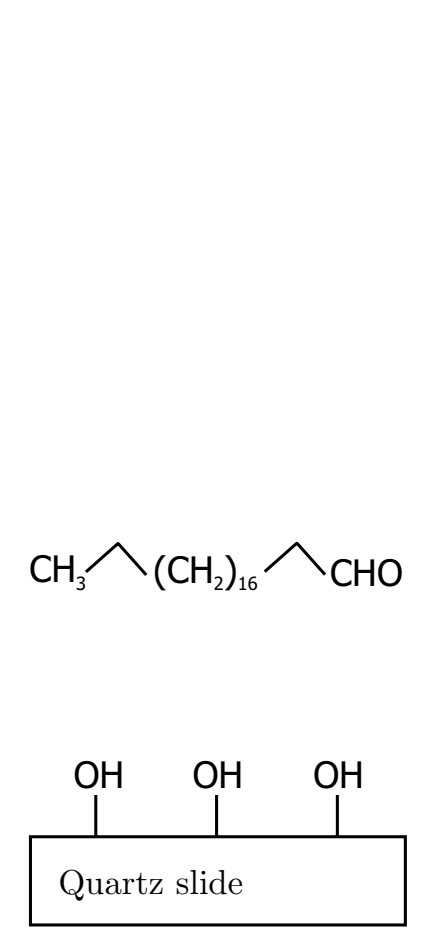

(a) Slide

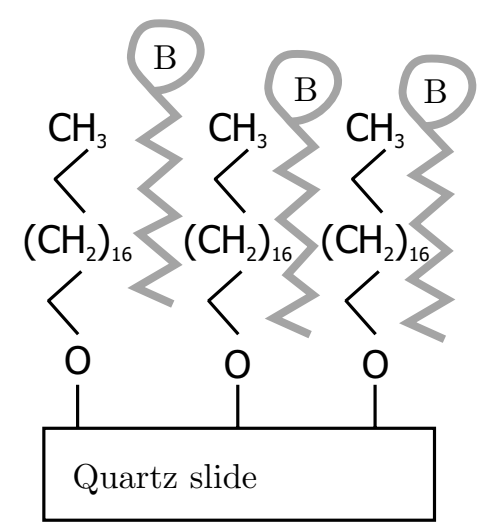

(b) Biotin attached

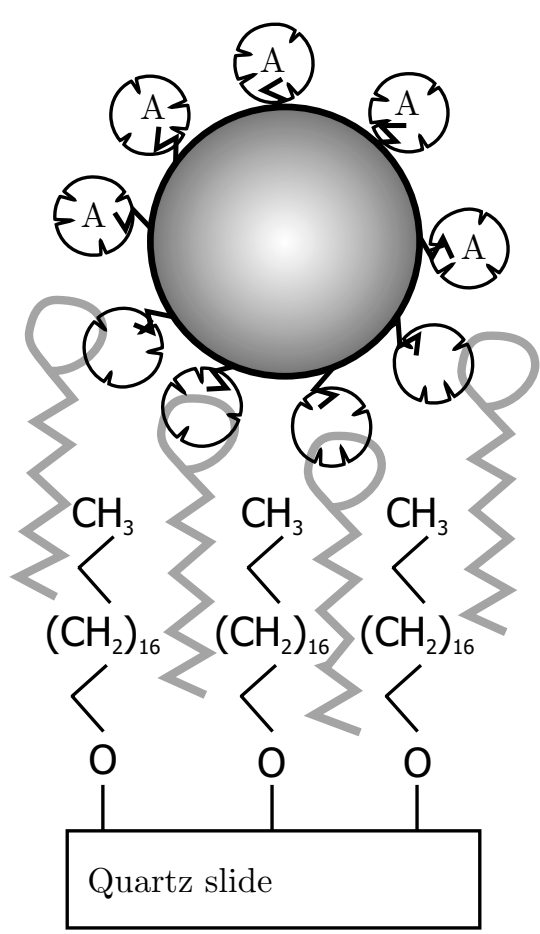

(c) Avidin on beads, with biotin attached

Figure 1. Modification of the quartz crystal surface by self-assembly octadecanol molecule. The hydrophobic moiety of octadecanol allows interaction with the biotin.

A beam splitter separates the laser beam into pump and probe pulses. The probe pulse illuminates an unbiased GaAs semiconductor emitter wafer to generate a $\mathrm{THz}$ beam, which is collimated and focused onto an electro-optic sampling crystal with paraboloidal mirrors. A pellicle after the second parabolic mirror allows the reference beam to travel collinearly with the $\mathrm{THz}$ wave through the electro-optic crystal $\langle 110\rangle$ ZnTe. A quarter wave plate (QWP), a Wollaston prism (P) and a pair of photodiodes are assembled for the balanced detection of the $\mathrm{THz}$ radiation. The reference and sample on quartz slides is mounted in a galvanometer and dithered in the $\mathrm{THz}$ beam at $10 \mathrm{~Hz}$ over a peak-to-peak distance of about $10 \mathrm{~mm}$.

\section{RESULTS}

By conjugating agarose particles to avidin and applying the conjugate to half of the quartz surface modified biotin, the $\mathrm{THz}$ difference signal between biotin and the biotin-avidin complex increases sharply. The two time-domain $\mathrm{THz}$ waveforms measured from the avidin target and the amplified avidin target are both larger than the signal from a non-specifically bound protein (DGDG - see below) and surface inhomogeneities. The waveforms are shown in Fig. 3. We are able to detect less than $10.3 \mathrm{ng} / \mathrm{cm}^{2}$ avidin on the quartz surface, indicating the potential capability of this newly developed method for future applications to detect DNA hybridization and antigen-antibody interactions.

Compared to the similar avidin-biotin experiment without agarose beads, an enhancement of eightfold has been observed. ${ }^{12}$ We believe that the signal enhancement is due to the increased refractive 


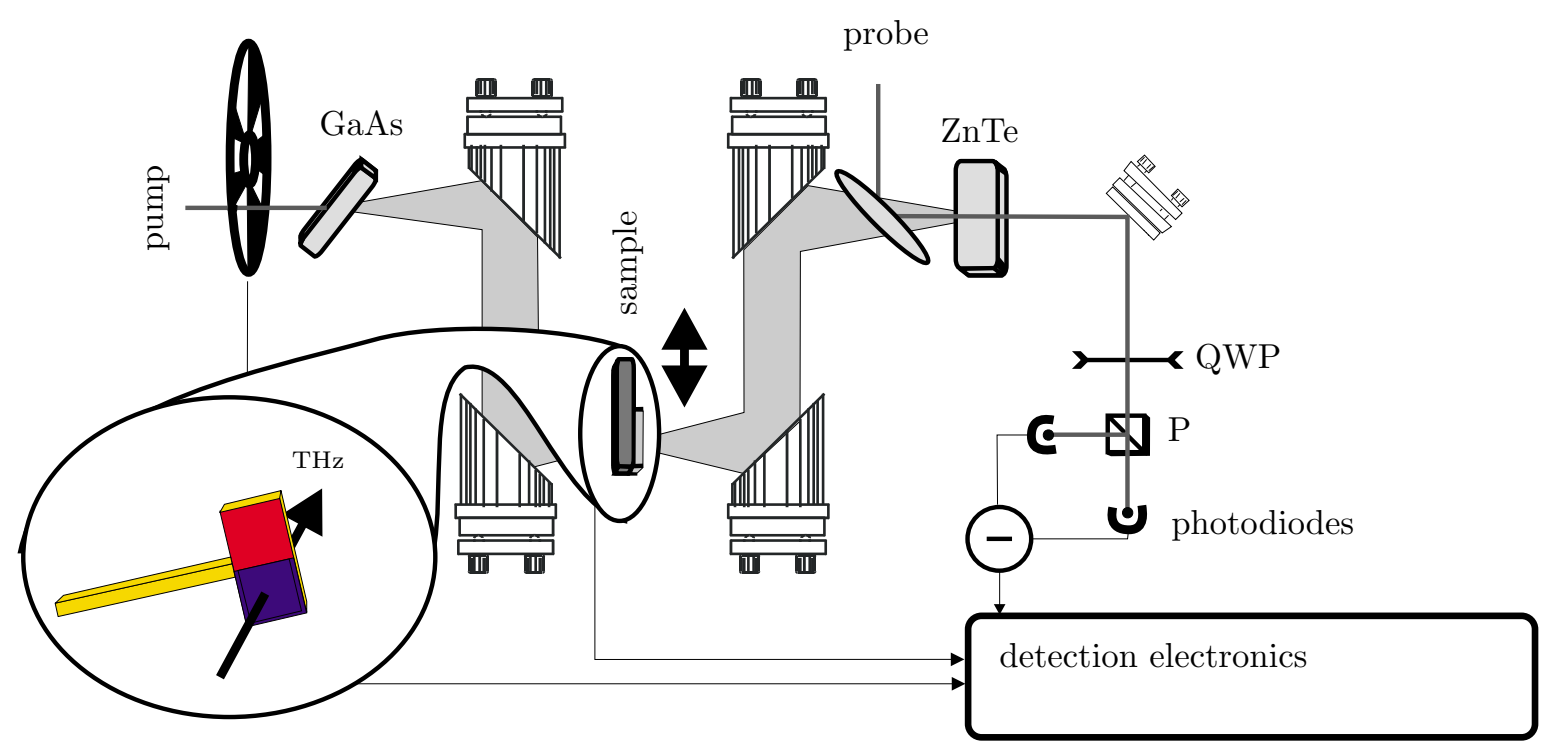

Figure 2. Terahertz biosensor system, showing an unbiased GaAs crystal THz emitter, and a $\langle 110\rangle \mathrm{ZnTe}$ crystal detector. The sample is dithered in and out of the $\mathrm{THz}$ beam by a galvanometer at a frequency of $10 \mathrm{~Hz}$. The $\mathrm{THz}$ beam is apertured to $2-\mathrm{mm}$ diameter, which corresponds to approximately $90 \%$ transmission. The travel distance of the sample slide is approximately $10 \mathrm{~mm}$ peak-to-peak, driven by a sine wave input. The detection electronics are used to demodulate both the 10- $\mathrm{Hz}$ sample modulation and suppress background noise (phase-sensitive detection). Although this system could be implemented in a number of ways, we use two lock-in amplifiers (Standford Research Systems, SR830 DSP LIAs). The time constant on the LIA used at $2 \mathrm{kHz}$ is $100-\mathrm{ms}$, which defines the detectors' noise bandwidth and thus our signal-to-noise ratio.

index of agarose particles, causing increased $\mathrm{THz}$ reflection at the biotin-avidin interface. This model is explored in Sect. 5. The repeatability of the waveforms shown in Fig. 3 is tested by measuring the DTDS signal at different lateral positions on each biosensor slide. The signal variation with position is $<10 \%$.

In order to test for the non-specific interaction of biotin towards avidin, and avoid false positive results, a control sample has been prepared. When a quartz crystal bearing biotin was incubated with a suspension of digalactosyldiacyl glycerol lipid (DGDG), instead of avidin-tagged agarose, the $\mathrm{THz}$ differential signal dropped by an order of magnitude, indicating that the previously observed $\mathrm{THz}$ signal was solely due to biotin-agarose conjugated avidin. The $\mathrm{THz}$ signal resulting from the biotin-DGDG interaction was presumably due to surface inhomogeneity. Although selectivity was not quantified in this report, we demonstrate that the specificity of biotin towards DGDG is far less than towards avidin. This is a significant result that exemplifies the use of molecular recognition as the primary source of biotin selectivity towards avidin.

\section{MODELLING}

A model of the biosensor system is critical for progressive system development and understanding of its limitations. In this paper we simulate our experimental results by modelling the biosensor slide as a series of homogeneous dielectric layers. The model construction is shown in Fig. 5. The THz radiation propagates through the layers, represented in Fig. 5 by the Fourier components of the sample and reference waveforms, $S_{s}$ and $S_{r}$, where $S_{s}=\mathcal{F} \mathcal{T}\left(y_{s}\right)$ and $S_{r}=\mathcal{F} \mathcal{T}\left(y_{r}\right)$. Expressions for $S_{s}$ and $S_{r}$ in terms of the incident $\mathrm{THz}$ radiation and the dielectric layers are developed in Sect. 5.1. 


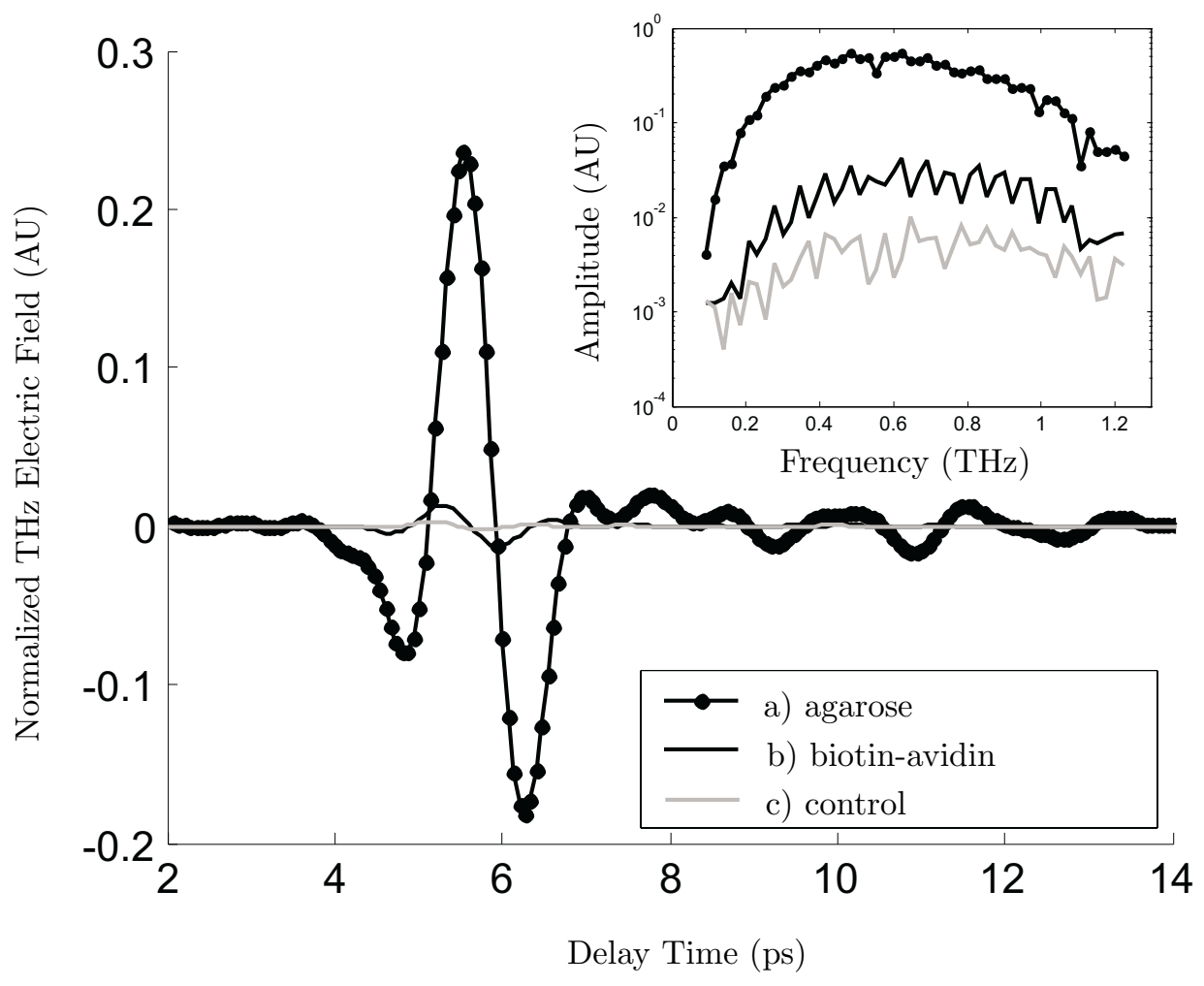

Figure 3. Time-domain $\mathrm{THz}$ pulses obtained by measuring the difference signal between (a) biotin and biotin-avidin conjugated agarose, (b) biotin and biotin-avidin complexes without beads, and (c) biotin and biotin-DGDG (control sample). These time-domain pulses have been smoothed numerically using a $0.1-1$ $\mathrm{THz}$ band-pass filter. The spectrometer signal-to-noise ratio in these measurements was greater than $10^{2}$ and the waveforms have been normalised to the peak of the $\mathrm{THz}$ reference waveform, $y_{r}$. The inset shows the waveforms in the Fourier domain (FFT). The intensity of the signal due to inhomogeneities and non-specific binding is less than the agarose and biotin-avidin signals.

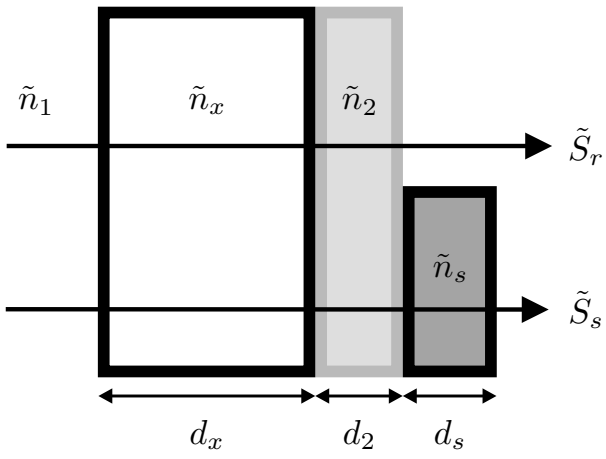

Figure 4. Notation used in DTDS simulation. $n_{x}$ is the quartz substrate, $n_{2}$ is the thin sensor layer (biotin) and $n_{s}$ is the thin target layer (avidin and conjugate avidin). The transmitted spectral components are the reference $S_{r}$ and the sample $S_{s}$. The surrounding medium, $n_{1}$, is air. 
The actual measured DTDS signal, as in Fig. 3, is represented by half the difference between $S_{s}$ and $S_{r}$, as discussed by Mickan et al. ${ }^{13}$ The biosensor is therefore modelled by the inverse Fourier transform of the difference between the reference and sample spectra. A simulated difference waveform is based on estimated material properties and the reference waveform $y_{r}$, which is measured using normal THz-TDS, as opposed to differential (DTDS). In normal THz-TDS, the sample is held stationary. The material properties are estimated to obtain a close fit to the experimental results. Section 5.2 shows that the simulated DTDS pulses fit very closely to the experimental results, thus validating our model.

\subsection{Propagation and transmission}

The propagation of a plane electro-magnetic wave through the dielectric layers shown in Fig. 5 can be modelled using Fresnel equations. ${ }^{14,15}$ Equation 1 is the transmission coefficient for a plane wave at normal incidence to a plane interface of two materials, passing from the dielectric of complex refractive index $\tilde{n}_{a}$ to that of $\tilde{n}_{b}$. Similarly, Eq. 2 is the reflection reflection coefficient. Equations $3 \& 4$ are versions of the propagation coefficient for a dielectric of thickness $d$.

$$
\begin{aligned}
t_{a b} & =\frac{2 \tilde{n}_{a}}{\tilde{n}_{a}+\tilde{n}_{b}}, \\
r_{a b} & =\frac{\tilde{n}_{a}-\tilde{n}_{b}}{\tilde{n}_{a}+\tilde{n}_{b}}, \\
p_{a} & =\exp \left(-j 2 \pi \tilde{n}_{a} d / \lambda\right), \\
& =\exp \left(-j 2 \pi n_{a} d / \lambda\right) \cdot \exp (-\alpha d / 2) .
\end{aligned}
$$

For thin layers, the transmission is modified by a Fabry-Pérot factor, $\operatorname{FP}(\omega)$, due to interference of multi-reflections within each layer. Equation 5 is the FP factor for a dielectric $\tilde{n}_{2}$, found between two dielectrics $\tilde{n}_{1}$ and $\tilde{n}_{3}$,

$$
\mathrm{FP}_{123} \approx \frac{1}{1-\left(\frac{\tilde{n}_{2}-\tilde{n}_{1}}{\tilde{n}_{2}+\tilde{n}_{1}}\right)\left(\frac{\tilde{n}_{2}-\tilde{n}_{3}}{\tilde{n}_{2}+\tilde{n}_{3}}\right) \cdot e^{-j 2 \tilde{n}_{2} \omega d_{2} / c_{0}}}
$$

DTDS measures time-domain pulses, thus the number of multi-reflections detected from each dielectric layer depends on the measurement duration and the thickness of each layer. For our biosensor experiments (Fig. 5), the quartz layer is thick enough so that no FP reflections are recorded. The biotin and avidin layers are sufficiently thin, $d \approx 1 \mu \mathrm{m}$, that influence from multi-reflections is small. Nevertheless, in the model calculations used, full FP expressions are included.

The spectral components of the signal and reference waveforms can be expressed in terms of a common term, $A(\omega)$, which represents common elements in the wave propagation, and the coefficients listed above:

$$
\begin{aligned}
& S_{r}=A(\omega) t_{21} p_{1} \mathrm{FP}_{x 21} \\
& S_{s}=A(\omega) t_{2 s} p_{s} t_{s 1} \mathrm{FP}_{x 2 s} \mathrm{FP}_{2 s 1}
\end{aligned}
$$

$A(\omega)$ represents effects in the system, including the characteristics of the emitter and detector, that are common to both the sample and detector. These effects are cancelled out in DTDS measurements.

\subsection{DTDS Modelling}

In DTDS, we measure the spectral components $S$ of the differential waveform, measured with high sensitivity by rapidly switching between the sample and reference,

$$
\begin{aligned}
& y_{d}=\left(y_{s}-y_{r}\right) / 2, \\
& S_{d}=\left(S_{s}-S_{r}\right) / 2 .
\end{aligned}
$$




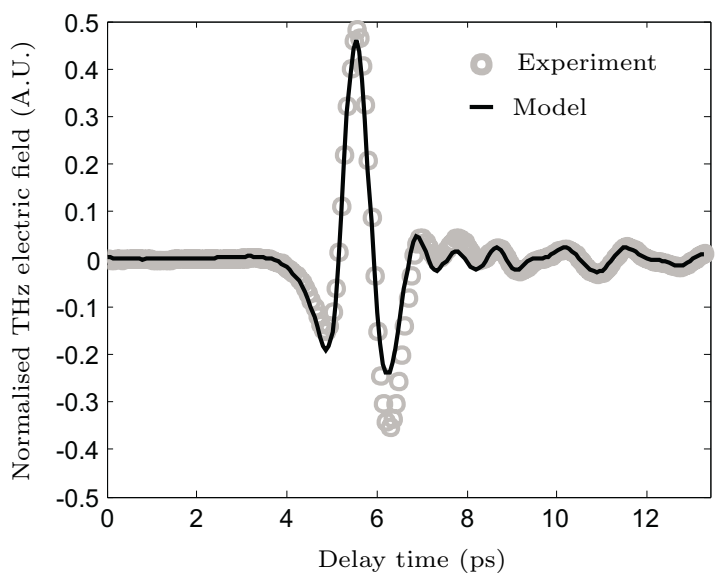

(a) Estimated parameters of the target layer (avidin conjugated to agarose beads): $d_{s}=$ $50 \mu \mathrm{m}, \tilde{n}_{s}=3-j 0.4$.

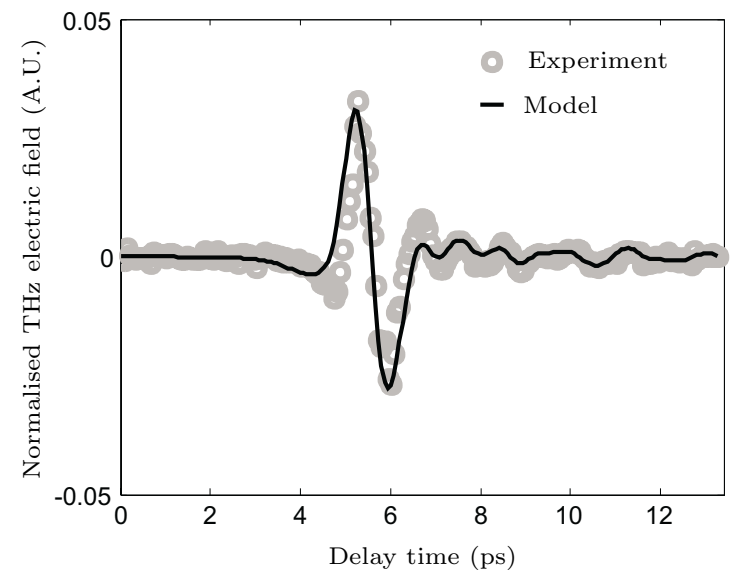

(b) Estimated parameters of the target layer (streptavidin): $d_{s}=5 \mu \mathrm{m}, \tilde{n}_{s}=1.1-j 0.0005$.

Figure 5. Simulated fits to the data shown in Fig. 3. The measured waveforms are normalized in amplitude to the peak of the THz reference pulse, $y_{r}$. The model waveforms are simulated using Eq. 10 with parameters set by the air, the quartz and the biotin layer. We approximate the refractive index and extinction coefficient values with values constant over the $\mathrm{THz}$ bandwidth. For the surrounding air, $n_{1}=1.0$ and $\kappa_{1}=0$. For the quartz substrate, $d_{x}=1 \mathrm{~mm}, n_{x}=2.1081$ and $\kappa_{x}=0.00032$. For the biotin sensor layer, $d_{2}=5 \mu \mathrm{m}$, $n_{2}=1.0001$ and $\kappa=0.001$. The estimated parameters of the different target layers are detailed in the sub-captions.

The factor of a half comes from the double-modulation detection scheme. ${ }^{13}$

To model the measured system, it is necessary to estimate the material parameters shown in Fig. 5. For our simple models, we assume the refractive index and extinction is constant across the frequency bandwidth of our spectrometer. This assumption of zero dispersion is a rough approximation, and accounts in part for the slight mismatch between the experimental and simulated results. In our experiments, the THz-frequency parameters for air, $\tilde{n}_{1}$, and the substrate, $\tilde{n}_{x}$, are known from literature and past experiments. For air, $\tilde{n}=1-j 0.0$ and for quartz, ${ }^{16} \tilde{n}=2.1081-j 0.00032$. The remaining parameters relate to the sensor layer (biotin) and the target layer (avidin or conjugated avidin).

The differential waveform is modelled by substituting the expressions for $S_{s}$ and $S_{r}$, Eqs. $7 \& 6$, into the difference Eq. 8. The simulated waveform, as shown in Fig. 5, is determined with an inverse Fourier transform.

$$
\begin{aligned}
S_{d} & =S_{r}\left(\frac{t_{2 s} p_{s} t_{s 1} \mathrm{FP}_{x 2 s} \mathrm{FP}_{2 s 1}}{t_{21} p_{1} \mathrm{FP}_{x 21}}-1\right) / 2, \\
y_{d \text { simulated }} & =\mathcal{F} \mathcal{T}^{-1}\left(S_{d}\right) .
\end{aligned}
$$

The THz refractive index of a thin biotin layer was estimated using standard DTDS of the biotin deposited on half a quartz slide, the other half of which was blank. The thickness of the biotin film was measured using atomic force microscopy (AFM) and found to be approximately $5 \mu \mathrm{m}$. The refractive index of the biotin film was then estimated by fitting a differential waveform, $y_{d}$, to the experimental data. $\tilde{n}_{2}$ was estimated to be approximately equal to air, $\tilde{n}_{\text {biotin }} \approx 1.001-j 0.001$. This is reasonable for a primarily non-polar molecule such as biotin; non-polar materials have a low THz absorption due to their low collective dipole moment. 
The refractive index of the target layer is the main free parameter for fitting the simulated waveform to the measured waveform. For the simulated fits shown in Fig. 5, the refractive index of avidin was taken to be approximately $\tilde{n}_{s}=1.1-j 0.0005$, and of the conjugated avidin and agarose, $\tilde{n}_{s}=3-j 0.4$. The conjugated avidin layer is expected to have a far greater thickness than the avidin alone, and the simulation of conjugated avidin fits with a layer thickness of $d_{s}=50 \mu \mathrm{m}$, which is far greater than the estimated thickness of the avidin layer alone, $d_{s}=5 \mu \mathrm{m}$.

The simulated DTDS waveforms approximate the experimental data well, as seen in Fig. 5. The estimated parameters for layer thickness and dielectric constant are reasonable for avidin and conjugated avidin, where a higher $\mathrm{THz}$ absorption and refractive index are expected for more polar molecules. It should be noted, however, that these simulations are intended primarily as a tool to understanding the mechanism of the biosensor's sensitivity, not for directly determining material properties. There is clearly some mismatch between the experiments and modelled results (Fig. 5), which is due to inaccuracies in the parameter estimations, inhomogeneities in the deposited films, and the assumption of a single refractive index value across the entire $\mathrm{THz}$ bandwidth, that is, a zero dispersion approximation. Nevertheless, the basic similarity in phase and amplitude indicates that the experimental signal is likely coming from $\mathrm{THz}$ transmission and reflection at the boundaries of the biotin and avidin dielectric layers. This indicates that further signal enhancements will be possible using greater dielectric mismatches. The noise from inhomogeneities can be reduced by using more laborious biofilm deposition techniques, such as spin-coating or Langmuir-Blodgett troughs.

\section{CONCLUSIONS}

The inherently high affinity of the avidin-biotin complementarity makes the $\mathrm{THz}$ biosensor attractive as an analytical device for a wide variety of biochemical studies. Owing to the technical difficulties encountered in the preparation of protein markers, the biotin-avidin system has proved of particularly broad application; almost any biologically active molecule can be tagged with biotin or avidin with only minimal effect on biological activity. Therefore using avidin-biotin technology together with high-refractive index beads, the DTDS biosensor will be able to play a pivotal role in detecting trace amounts of biological materials. Likewise antibody-antigen technology may also benefit from this new amplified $\mathrm{THz}$ detection method. Since antibodies can be produced for almost all classes of substances, for example, proteins, polysaccharides, nucleic acids, including more complex particles such as pollens, infectious agents, viruses and tissue cells, we expect that the enhanced DTDS system should be able to detect minute amount of a wide range of biomolecules. Further enhancement may be possible by conjugating target molecules to gold or carbon micro-spheres.

\section{Acknowledgements}

We would like to acknowledge support from the U.S. National Science Foundation, the U.S. Army Research Office, the Wadsworth Centre of the New York State Department of Health, the Australian Fulbright Commission and the Australian Research Council. We would like to thank D. Liu at Rensselaer for assistance with the AFM.

\section{REFERENCES}

1. V. Deckert, D. Zeisel, R. Zenobi, and T. Vo-Dinh, "Near-field surface-enhanced raman imaging of dye-labeled DNA with 100-nm resolution," Analytical Chemistry 70(13), pp. 2646-2650, 1998.

2. F. Regan, M. Meaney, J. G. Vos, B. D. MacCraith, and J. E. Walsh, "Determination of pesticides in water using ATR-FTIR spectroscopy on PVC/chloroparaffin coatings," Analytica Chimica Acta 334(1-2), pp. 85-92, 1996.

3. R. Kalsson and R. Stahlberg Analytical Chemistry 228, p. 274, 1995.

4. X. Song and B. I. Swanson, "Direct, ultrasensitive, and selective optical detection of protein toxins using multivalent interactions," Analytical Chemistry 71(11), pp. 2097-2107, 1999. 
5. A. Menikh, R. MacColl, C. A. Mannella, and X.-C. Zhang, "Terahertz biosensing technology: Frontiers and progress," ChemPhysChem 3(8), pp. 655-658, 2002.

6. M. Nagel, P. Haring Bolívar, M. Brucherseifer, H. Kurz, A. Bosserhoff, and R. Büttner, "Integrated THz technology for label-free genetic diagnostics," Applied Physics Letters 80(1), pp. 154156, 2002.

7. M. Nagel, P. Haring Bolívar, M. Brucherseifer, H. Kurz, A. Bosserhoff, and R. Büttner, "Integrated planar terahertz resonators for femtomolar sensitivity label-free detection of DNA hybridization," Applied Optics 41(10), pp. 2074-2078, 2002.

8. S. P. Mickan, A. Menikh, H. Liu, C. A. Mannella, D. Abbott, J. Munch, and X.-C. Zhang, "Tray-based sensing of thin film lipid-protein interactions," Physics in Medicine and Biology In Press, 2002.

9. N. M. Green, "Avidin," Advances in Protein Chemistry 29, pp. 85-133, 1975.

10. P. Cuatrecasas and M. Wilchek, "Single-step purification of avidin from egg white by affinity chromatography on biocytin-Sepharose columns," Biochemical and Biophysical Research Communications 33(2), pp. 235-239, 1968.

11. M. Wilchek and E. A. Bayer, "The avidin-biotin complex and bioanalytical applications," Analytical Biochemistry 171(1), pp. 1-32, 1988.

12. A. Menikh, S. P. Mickan, R. MacColl, C. A. Mannella, and X.-C. Zhang, "Amplified detection of avidin-biotin binding using terahertz wave technology," Chemical Physics Letters Submitted, 2002.

13. S. P. Mickan, K. S. Lee, T.-M. Lu, J. Munch, D. Abbott, and X.-C. Zhang, "Double modulated differential THz-TDS for thin film dielectric characterization," Microelectronics Journal In Press, 2002.

14. M. Born and E. Wolf, Principles of Optics, Cambridge University Press, 7th ed., 1999.

15. L. Duvillaret, F. Garet, and J.-L. Coutaz, "A reliable method for extraction of material parameters in terahertz time-domain spectroscopy," IEEE Journal of Selected Topics in Quantum Electronics 2(3), pp. 739-746, 1996.

16. D. Grischkowsky, S. Keiding, M. van Exter, and C. Fattinger, "Far-infrared time-domain spectroscopy with terahertz beams of dielectrics and semiconductors," Journal of the Optical Society of America B: Optical Physics 7(10), pp. 2006-2015, 1990. 